\title{
ODD RIEMANNIAN SYMMETRIC SPACES ASSOCIATED TO FOUR-FORMS
}

\author{
VICENTE CORTÉS*
}

\begin{abstract}
To any four-form $\Omega$ satisfying certain system of quadratic equations a Lie superalgebra $\mathfrak{g}=$ $\mathfrak{g}_{0}+\mathfrak{g}_{1}$ endowed with an invariant scalar superproduct is associated. The geometric structure of the corresponding odd symmetric superspace, which is of hyper-Kähler type, is described. Finally, some classes of solutions to the quadratic system are discussed.
\end{abstract}

\section{Introduction}

Hyper-Kähler manifolds are Riemannian manifolds with holonomy group in $\operatorname{Sp}(n) \subset \mathrm{SO}(4 n)$. As examples of Ricci-flat Einstein manifolds, they are particularly interesting but not easy to construct [4]. It is natural to extend the above definition to the case when the manifold is endowed with a pseudo-Riemannian (i.e possibly indefinite) metric, as follows: a pseudo-hyper-Kähler manifold is a pseudo-Riemannian manifold with holonomy in $\operatorname{Sp}(k, l) \subset \mathrm{SO}(4 k, 4 l)$. There is an other natural extension of hyper-Kähler manifolds from the Riemannian to the pseudo-Riemannian setting: a para-hyper-Kähler manifold is a pseudo-Riemannian manifold with holonomy in the real symplectic group $\mathrm{Id}_{\mathrm{R}^{2}} \otimes \mathrm{Sp}\left(\mathrm{R}^{2 n}\right) \subset \mathrm{GL}\left(\mathrm{R}^{2} \otimes \mathrm{R}^{2 n}\right)$, which is a subgroup of $\mathrm{SO}(2 n, 2 n)$. These two types of constraints on the holonomy of a pseudo-Riemannian manifold correspond to the different real forms, $\operatorname{Sp}(k, l)$ and $\operatorname{Sp}\left(\mathrm{R}^{2 n}\right)$, of the complex symplectic group $\operatorname{Sp}\left(\mathrm{C}^{2 n}\right)$.

It was found in [1], [2] that non-flat pseudo-Riemannnian symmetric spaces with pseudo-hyper-Kählerian and para-hyper-Kählerian holonomy exist in abundance. They all have solvable holonomy group and correspond to solutions of a system of homogeneous quadratic equations for a quartic tensor $S$, see (1) below.

The aim of this paper is to extend this correspondence to the category of supermanifolds. The role of the quartic tensor $S$ will be played by a four-form $\Omega$ satisfying a new system of quadratic equations (2).

\footnotetext{
* This work was supported by the 'Schwerpunktprogramm Stringtheorie' of the Deutsche Forschungsgemeinschaft.

Received January 6, 2005.
} 
In the following, we state the results of [6], [1], [2] which we shall transpose to the category of supermanifolds. The main results of this paper will be briefly summarized at the end of the introduction.

Let $\left(E, \omega_{E}\right)$ and $\left(H, \omega_{H}\right)$ be (real or complex) symplectic vector spaces of dimension $2 n$ and 2 respectively. Given a quartic tensor $S \in S^{4} E$ and two vectors $e, e^{\prime} \in E$, the contraction of $S$ with $e e^{\prime} \in S^{2} E$, by means of $\omega_{E}$, is denoted $S_{e e^{\prime}} \in S^{2} E$. We can consider the following system of quadratic equations:

$$
S_{e e^{\prime}} \cdot S=0 \quad \text { for all } e, e^{\prime} \in E,
$$

where the dot stands for the action of the symplectic Lie algebra $\mathfrak{s p}(E) \cong S^{2} E$ on the $\mathfrak{s p}(E)$-module $S^{4} E$.

The following theorem was proven in [1].

THeOREM 1. Any $S \in S^{4} E$ satisfying the equation (1) defines a Lie algebra $\mathfrak{g}$ endowed with a $\mathrm{Z} / 2 \mathrm{Z}$-grading $\mathfrak{g}=\mathfrak{g}_{0}+\mathfrak{g}_{1}$ of Lie algebras. More precisely,

$$
\begin{aligned}
& \mathfrak{g}_{0}:=S_{E E}:=\operatorname{span}\left\{S_{e e^{\prime}} \mid e, e^{\prime} \in E\right\} \subset S^{2} E \cong \mathfrak{s p}(E), \\
& \mathfrak{g}_{1}:=E \otimes H,
\end{aligned}
$$

with $\mathfrak{g}_{0}$ acting via the identification $\mathfrak{s p}(E) \cong \mathfrak{s p}(E) \otimes \operatorname{Id}_{H} \subset \mathfrak{g}(E \otimes H)$ and

$$
\left[e \otimes h, e^{\prime} \otimes h^{\prime}\right]:=\omega_{H}\left(h, h^{\prime}\right) S_{e e^{\prime}} .
$$

Let $G$ be the simply connected Lie group with Lie algebra $g$ and $K \subset G$ the closed connected Lie subgroup with Lie algebra $K=\mathfrak{g}_{0}$. Then $M_{S}=G / K$ is a simply connected symmetric space. The scalar product $\omega_{E} \otimes \omega_{H}$ induces on $M$ a $G$-invariant pseudo-Riemannian metric if the ground field $\mathrm{K}$ is $\mathrm{R}$ and a $G$-invariant complex (i.e. holomorphic) Riemannian metric if $\mathrm{K}=\mathrm{C}$. More precisely, we have the following results:

Theorem 2 ([1]). Let $\mathrm{K}=\mathrm{C}$. Any complex Riemannian symmetric space $M_{S}=G / K$ associated to $S \in S^{4} E$ satisfying equation (1) admits a $G$ invariant complex hyper-Kähler structure and any simply connected symmetric complex hyper-Kähler manifold is of this form.

Moreover, it was proven in [1] that the complex manifold $M_{S}$ admits a real form which is a symmetric pseudo-hyper-Kähler manifold, provided that $S$ satisfies a suitable reality condition. The reality condition is defined using a quaternionic structure $j_{E}$ on $E$ which satisfies $j_{E}^{*} \omega_{E}=\bar{\omega}_{E}$. 
TheOREM 3 ([2]). Let $\mathrm{K}=\mathrm{R}$. Any pseudo-Riemannian symmetric space $M_{S}=G / K$ associated to $S \in S^{4} E$ satisfying equation (1) admits a $G$ invariant para-hyper-Kähler structure. Conversely, any simply connected symmetric para-hyper-Kähler manifold is of this form.

It is shown in [1], [2] that any tensor $S \in S^{4} E_{0} \subset S^{4} E$ over an isotropic subspace $E_{0} \subset\left(E, \omega_{E}\right)$ is a solution of (1) and defines a complex hyperKähler (respectively, a para-hyper-Kähler) symmetric space $M_{S}$ with Abelian holonomy group if $K=C$ (respectively, if $K=R$ ). In the case $K=C$, the complex manifold $M_{S}$ admits a real form which is a symmetric pseudo-hyperKähler manifold with Abelian holonomy group, provided that $S$ satisfies a suitable reality condition. Conversely, any complex hyper-Kähler, para-hyperKähler or pseudo-hyper-Kähler symmetric space with Abelian holonomy group is obtained in this way, see Theorem 10.

The problem of determining the general solution of the quadratic system (1) is open, despite the attempt of [1] to prove that any solution is a quartic tensor over an isotropic subspace, which is inconclusive due to a sign mistake in the calculations. Pseudo-hyper-Kähler symmetric spaces with non-Abelian holonomy group were recently constructed by Kath and Olbrich [7]. They correspond to solutions of (1), for which there is no isotropic subspace $E_{0}$ such that $S \in S^{4} E_{0} \subset S^{4} E$.

The following result is due to Cahen and Parker [6]:

THEOREM 4. Let $\mathrm{g}=\mathfrak{g}_{0}+\mathfrak{g}_{1}$ be a $\mathrm{Z} / 2 \mathrm{Z}$-graded Lie algebra such that $\mathfrak{g}_{0}=\left[\mathfrak{g}_{1}, \mathfrak{g}_{1}\right]$. If $\mathfrak{g}_{1}$ is endowed with an $\operatorname{ad}_{\mathfrak{g}_{0}}$-invariant scalar product $\langle\cdot, \cdot\rangle_{1}$ then there exists a unique $\mathrm{ad}_{\mathfrak{g}_{0}}$-invariant scalar product $\langle\cdot, \cdot\rangle_{0}$ on $\mathfrak{g}_{0}$ which extends $\langle\cdot, \cdot\rangle_{1}$ to an $\operatorname{ad}_{\mathfrak{g}}$-invariant scalar product $\langle\cdot, \cdot\rangle=\langle\cdot, \cdot\rangle_{0} \oplus\langle\cdot, \cdot\rangle_{1}$ on $\mathfrak{g}$.

As a corollary, we obtain

Corollary 1. Let $\mathfrak{g}=\mathfrak{g}_{0}+\mathfrak{g}_{1}$ be the Lie algebra defined by a quartic tensor $S \in S^{4} E$ satisfying the equation (1) and $K \subset G$ two connected Lie groups with Lie $G=\mathfrak{g}$ and Lie $K=\mathfrak{f}:=\mathfrak{g}_{0}$. Then there exists a unique $\operatorname{Ad}_{K}$-invariant scalar product $\langle\cdot, \cdot\rangle_{0}$ on $\mathrm{g}_{0}$ which extends the $\operatorname{Ad}_{K}$-invariant scalar product $\langle\cdot, \cdot\rangle_{1}:=\omega_{E} \otimes \omega_{H}$ on $\mathfrak{g}_{1}=E \otimes H$ to an $\operatorname{Ad}_{G}$-invariant scalar product $\langle\cdot, \cdot\rangle=\langle\cdot, \cdot\rangle_{0} \oplus\langle\cdot, \cdot\rangle_{1}$ on g. It is defined by

$$
\left\langle S_{e e^{\prime}}, S_{e^{\prime \prime} e^{\prime \prime \prime}}\right\rangle_{0}:=\omega_{E}\left(S_{e e^{\prime}} e^{\prime \prime}, e^{\prime \prime \prime}\right) \quad \text { for all } e, e^{\prime}, e^{\prime \prime}, e^{\prime \prime \prime} \in E .
$$

Proof. Since $G$ is connected, the $\operatorname{Ad}_{G}$-invariance of the scalar product is equivalent to the $\mathrm{ad}_{\mathfrak{g}}$-invariance, i.e. to:

$$
\langle[X, Y], Z\rangle=-\langle Y,[X, Z]\rangle
$$


for all elements $X, Y$ and $Z$ of $g$. Evaluating this for $X=e \otimes h, Y=e^{\prime} \otimes h^{\prime} \in$ $\mathfrak{g}_{1}=E \otimes H$ and $Z=S_{e^{\prime \prime} e^{\prime \prime \prime}} \in \mathfrak{g}_{0}=S_{E E}$, we get

$$
\begin{aligned}
\omega_{H}\left(h, h^{\prime}\right)\left\langle S_{e e^{\prime}}, S_{e^{\prime \prime} e^{\prime \prime \prime}}\right\rangle_{0} & =\left\langle e^{\prime} \otimes h^{\prime}, S_{e^{\prime \prime}} e^{\prime \prime \prime} e \otimes h\right\rangle_{1} \\
& =-\omega_{H}\left(h, h^{\prime}\right) \omega_{E}\left(e^{\prime}, S_{e^{\prime \prime} e^{\prime \prime \prime}} e\right) \\
& =\omega_{H}\left(h, h^{\prime}\right) \omega_{E}\left(S_{e e^{\prime}} e^{\prime \prime}, e^{\prime \prime \prime}\right) .
\end{aligned}
$$

This establishes the explicit formula for the scalar product.

Lie algebras admitting an ad-invariant scalar product and Lie superalgebras admitting an ad-invariant scalar superproduct have been much studied in the literature, see e.g. [11], [5], [8], [3] and [9]. They play some role in BRST quantization, see e.g. [10].

The main purpose of this paper is to introduce and study an interesting class of Lie superalgebras $\mathfrak{g}=\mathfrak{g}(\Omega)=\mathfrak{g}_{0}+\mathfrak{g}_{1}$ which are associated to certain four-forms $\Omega$ and admit an ad-invariant scalar superproduct. The basic data for the construction are the same as above, with the difference that, instead of a symplectic vector space $\left(E, \omega_{E}\right)$, we will now consider a vector space $\left(E, g_{E}\right)$ of arbitrary dimension $n$ endowed with a scalar product $g_{E}$ (i.e. a nondegenerate symmetric bilinear form on $E$ ). The role of the quartic symmetric tensor $S$ is now played by a four-form $\Omega \in \wedge^{4} E \cong \wedge^{4} E^{*}$ satisfying the following system of quadratic equations:

$$
\Omega_{e \wedge e^{\prime}} \cdot \Omega=0 \quad \text { for all } e, e^{\prime} \in E,
$$

where $\Omega_{e \wedge e^{\prime}} \in \wedge^{2} E$ stands for the contraction of $\Omega$ with $e \wedge e^{\prime}$ by means of $g_{E}$, the dot stands for the action of the orthogonal Lie algebra $\mathfrak{S} \mathfrak{D}(E) \cong \wedge^{2} E$ on the $\mathfrak{s} \mathfrak{D}(E)$-module $\wedge^{4} E$.

Analogues in the category of supermanifolds of the above Theorems 1, 2, 3 and 4 are established below in Theorems 5, 7, 8 and 6, respectively. Moreover, some classes of solutions of the equation (2) are discussed. In particular, all solutions for which the Lie subalgebra $\Omega_{E \wedge E} \subset \mathfrak{S D}(E)$ is Abelian are determined, see Theorem 9.

I thank Dmitri Alekseevsky, Saïd Benayadi, Lionel Bérard Bergery, and Francisco Martín Cabrera for useful discussions. 


\section{Lie superalgebras associated to four-forms}

Let $E$ be a $\mathrm{K}$-vector space of dimension $n$ and $g_{E}$ a scalar product on $E$, i.e. a nondegenerate symmetric bilinear form.

Theorem 5. Any $\Omega \in \wedge^{4} E$ satisfying the equation (2) defines a Lie superalgebra $\mathfrak{g}=\mathfrak{g}(\Omega)=\mathfrak{g}_{0}+\mathfrak{g}_{1}$. More precisely,

$$
\begin{aligned}
& \mathfrak{g}_{0}:=\Omega_{E \wedge E}:=\operatorname{span}\left\{\Omega_{e \wedge e^{\prime}} \mid e, e^{\prime} \in E\right\} \subset \wedge^{2} E \cong \mathfrak{g} \mathfrak{D}(E), \\
& \mathfrak{g}_{1}:=E \otimes H,
\end{aligned}
$$

with $\mathrm{g}_{0}$ acting via the identification $\mathfrak{S} \mathfrak{D}(E) \cong \mathfrak{S} \mathfrak{D}(E) \otimes \operatorname{Id}_{H} \subset \mathfrak{g l}(E \otimes H)$ and

$$
\left[e \otimes h, e^{\prime} \otimes h^{\prime}\right]:=\omega_{H}\left(h, h^{\prime}\right) \Omega_{e \wedge e^{\prime}} .
$$

Proof. We have to check that, with the above definitions, the Jacobi identity is satisfied. Using equation (2), we first check that $\mathfrak{g}_{0} \subset \mathfrak{S} \mathfrak{D}(E)$ is a Lie subalgebra:

$$
\begin{aligned}
& 0=\left(\Omega_{e \wedge e^{\prime}} \cdot \Omega\right)_{e^{\prime \prime} \wedge e^{\prime \prime \prime}}
\end{aligned}
$$

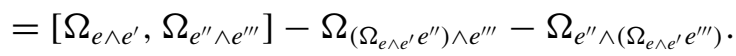

This shows that $\left[\Omega_{e \wedge e^{\prime}}, \Omega_{e^{\prime \prime} \wedge e^{\prime \prime \prime}}\right] \in \Omega_{E \wedge E}$ for all $e, e^{\prime}, e^{\prime \prime}, e^{\prime \prime \prime} \in E$ and, hence, that $\mathrm{g}_{0} \subset \mathfrak{S} \mathfrak{D}(E)$ is a Lie subalgebra. In particular, the Jacobi identity is satisfied for $X, Y, Z \in \mathfrak{g}_{0}$. It is also satisfied for $X, Y \in \mathfrak{g}_{0}$ and $Z \in \mathfrak{g}_{1}$, since $\mathfrak{g}_{1}$ is a $\mathrm{g}_{0}$-module. So we are left with two cases.

Case 1: $X=\Omega_{e^{\prime \prime} \wedge e^{\prime \prime \prime}} \in \mathfrak{g}_{0}$ and $Y=e \otimes h, Z=e^{\prime} \otimes h^{\prime} \in \mathfrak{g}_{1}=E \otimes H$. Using equation (2), we obtain:

$$
\begin{aligned}
& {[X,[Y, Z]]-[[X, Y], Z]-[Y,[X, Z]]} \\
& =\omega_{H}\left(h, h^{\prime}\right)\left(\left[\Omega_{e^{\prime \prime} \wedge e^{\prime \prime \prime}}, \Omega_{e \wedge e^{\prime}}\right]-\Omega_{\left(\Omega_{\left.e^{\prime \prime} \wedge e^{\prime \prime \prime} e\right) \wedge e^{\prime}}\right.}-\Omega_{e \wedge\left(\Omega_{e^{\prime \prime} \wedge e^{\prime \prime \prime} e^{\prime}}\right)}\right) \\
& =\omega_{H}\left(h, h^{\prime}\right)\left(\Omega_{e^{\prime \prime} \wedge e^{\prime \prime \prime}} \cdot \Omega\right)_{e \wedge e^{\prime}}=0 .
\end{aligned}
$$

Case 2: $X=e \otimes h, Y=e^{\prime} \otimes h^{\prime}, Z=e^{\prime \prime} \otimes h^{\prime \prime} \in \mathfrak{g}_{1}=E \otimes H$. Using the skew-symmetry of $\Omega \in \wedge^{4} E$ in the first three arguments, we get:

$$
\begin{aligned}
{[X,[Y, Z]]-[[X, Y], Z]+[Y,[X, Z]] } & \\
= & -\omega_{H}\left(h^{\prime}, h^{\prime \prime}\right) \Omega_{e^{\prime} \wedge e^{\prime \prime}} \otimes h-\omega_{H}\left(h, h^{\prime}\right) \Omega_{e \wedge e^{\prime} e^{\prime \prime}} \otimes h^{\prime \prime} \\
& \quad-\omega_{H}\left(h, h^{\prime \prime}\right) \Omega_{e \wedge e^{\prime \prime}} e^{\prime} \otimes h^{\prime} \\
= & -\Omega_{e \wedge e^{\prime}} e^{\prime \prime} \otimes\left(\omega_{H}\left(h, h^{\prime}\right) h^{\prime \prime}+\omega_{H}\left(h^{\prime}, h^{\prime \prime}\right) h+\omega_{H}\left(h^{\prime \prime}, h\right) h^{\prime}\right) .
\end{aligned}
$$


Now it is sufficient check that

$$
\omega_{H}\left(h, h^{\prime}\right) h^{\prime \prime}+\omega_{H}\left(h^{\prime}, h^{\prime \prime}\right) h+\omega_{H}\left(h^{\prime \prime}, h\right) h^{\prime}=0
$$

for all $h, h^{\prime}, h^{\prime \prime} \in H$. This is obvious, since this cyclic sum is completely skew in $\left(h, h^{\prime}, h^{\prime \prime}\right)$ and $\operatorname{dim} H=2$.

The above calculation shows that $\operatorname{ad}_{X}: Y \rightarrow[X, Y]$ is a superderivation for all $X \in \mathfrak{g}$. This is the content of the Jacobi identity.

\section{Invariant scalar superproducts on a class of Lie superalgebras}

Definition 1. Let $V=V_{0} \oplus V_{1}$ be a Z/2Z-graded $\mathrm{K}$-vector space. The elements of $V_{0} \cup V_{1}$ are called homogeneous elements. The degree $\alpha \in Z / 2 Z$ of $X \in V_{\alpha}$ is denoted by $\widetilde{X}$. A K-bilinear form $\langle\cdot, \cdot\rangle$ on $V$ is called even if $\left\langle V_{0}, V_{1}\right\rangle=\left\langle V_{1}, V_{0}\right\rangle=0$. An even K-bilinear form $\langle\cdot, \cdot\rangle$ on $V$ and is called supersymmetric if

$$
\langle X, Y\rangle=(-1)^{\tilde{X}} \tilde{Y}\langle Y, X\rangle
$$

for all homogeneous elements $X, Y \in V$. A scalar superproduct, more precisely, an even scalar superproduct, on $V$ is a nondegenerate even supersymmetric K-bilinear form $\langle\cdot, \cdot\rangle$ on $V$.

In other words, a scalar superproduct is a direct $\operatorname{sum}\langle\cdot, \cdot\rangle=\langle\cdot, \cdot\rangle_{0} \oplus\langle\cdot, \cdot\rangle_{1}$ of a (possibly indefinite) scalar product $\langle\cdot, \cdot\rangle_{0}$ on $V_{0}$ and a symplectic bilinear form $\langle\cdot, \cdot\rangle_{1}$ on the ungraded vector space underlying $V_{1}$.

Definition 2. Let $\mathfrak{g}=\mathfrak{g}_{0}+\mathfrak{g}_{1}$ be a Lie superalgebra. A symmetric decomposition (or Z/2Z-grading) of $\mathfrak{g}$ is a direct decomposition $\mathfrak{g}=\mathfrak{f}+\mathfrak{m}$ of $\mathrm{Z} / 2 \mathrm{Z}$-graded vector spaces such that

$$
[\mathfrak{f}, \mathfrak{f}] \subset \mathfrak{f}, \quad[\mathfrak{f}, \mathfrak{m}] \subset \mathfrak{m} \quad \text { and } \quad[\mathfrak{m}, \mathfrak{m}] \subset \mathfrak{f} .
$$

Here we prove the following generalization of Theorem 4:

THeOREM 6. Let $\mathfrak{g}=\mathfrak{f}+\mathfrak{m}$ be a symmetric decomposition of a Lie superalgebra $\mathfrak{g}=\mathfrak{g}_{0}+\mathfrak{g}_{1}$ such that $\mathfrak{f}=[\mathfrak{m}, \mathfrak{m}]$. If $\mathfrak{m}$ is endowed with an $\operatorname{ad}_{\mathfrak{f}}$ invariant scalar superproduct $\langle\cdot, \cdot\rangle_{\mathfrak{m}}$ then there exists a unique $\operatorname{ad}_{\mathfrak{f}}$-invariant scalar superproduct $\langle\cdot, \cdot\rangle_{\mathfrak{f}}$ on $\mathfrak{f}$ which extends $\langle\cdot, \cdot\rangle_{\mathfrak{m}}$ to an $\operatorname{ad}_{\mathfrak{g}}$-invariant scalar superproduct $\langle\cdot, \cdot\rangle=\langle\cdot, \cdot\rangle_{\mathfrak{f}} \oplus\langle\cdot, \cdot\rangle_{\mathfrak{m}}$ on $\mathrm{g}$.

Proof. The $\mathrm{ad}_{\mathfrak{g}}$-invariance of the scalar superproduct $\langle\cdot, \cdot\rangle$ means that

$$
\langle[X, Y], Z\rangle=-(-1)^{\tilde{X} \widetilde{Y}}\langle Y,[X, Z]\rangle
$$


for all homogeneous elements $X, Y, Z \in \mathfrak{g}$. Now let $X, Y, Z, W \in \mathfrak{m}$ and put $A=[X, Y]$ and $B=[Z, W] \in \mathfrak{f}=[\mathfrak{m}, \mathfrak{m}]$. The $a_{\mathfrak{g}}$-invariance of $\langle\cdot, \cdot\rangle=\langle\cdot, \cdot\rangle_{\mathfrak{f}} \oplus\langle\cdot, \cdot\rangle_{\mathfrak{m}}$ implies that

$$
\langle A, B\rangle_{\mathfrak{f}}=-(-1)^{\tilde{X} \widetilde{Y}}\langle Y,[X, B]\rangle_{\mathfrak{m}} .
$$

This proves the uniqueness. To prove the existence we have to prove that the right-hand side of (4) depends only on $A$ and $B$ and check the full $\mathrm{ad}_{\mathfrak{g}^{-}}$ invariance. It is clear that the right-hand side of (4) depends on $Z, W \in \mathfrak{m}$ only through $B=[Z, W]$. Similarly, it depends on $X, Y \in \mathfrak{m}$ only through $A=[X, Y]$, in virtue of the identity

$$
(-1)^{\tilde{X} \widetilde{Y}}\langle Y,[X, B]\rangle_{\mathfrak{m}}=(-1)^{\tilde{Z} \widetilde{W}}\langle[A, W], Z\rangle_{\mathfrak{m}},
$$

which follows from the next lemma. To prove this identity we first remark that

$$
\mathfrak{m} \times \mathfrak{m} \ni(X, Y) \mapsto R(X, Y):=-\left.\operatorname{ad}_{[X, Y]}\right|_{\mathfrak{m}} \in \mathfrak{b} \mathfrak{s} \mathfrak{p}(\mathfrak{m})
$$

defines an algebraic curvature tensor $R$ of type $\mathfrak{b} \mathfrak{s p}(\mathfrak{m})$, i.e. a skew-symmetric (in the super sense) bilinear form on the supervector space $\mathfrak{m}$ with values in the orthosymplectic Lie superalgebra $\mathfrak{D} \mathfrak{g} \mathfrak{p}(\mathfrak{m})$ which satisfies the first Bianchi identity:

$$
R(X, Y) Z+(-1)^{\widetilde{X}(\widetilde{Y}+\widetilde{Z})} R(Y, Z) X+(-1)^{\widetilde{Z}(\widetilde{X}+\widetilde{Y})} R(Z, X) Y=0
$$

for all $X, Y, Z \in \mathfrak{m}$.

Proposition 1. Let $\mathfrak{g}=\mathfrak{f}+\mathfrak{m}$ be a symmetric decomposition of a Lie superalgebra $\mathfrak{g}$ and $R: \mathfrak{m} \times \mathfrak{m} \rightarrow \mathfrak{D} \mathfrak{s p}(\mathfrak{m})$ the bilinear map defined by (6). Then $R$ is an $\mathrm{ad}_{\mathfrak{f}}$-invariant algebraic curvature tensor $R$ of type $\mathfrak{D} \mathfrak{S p}(\mathfrak{m})$.

Proof. The Bianchi identity and $\mathrm{ad}_{\mathrm{f}}$-invariance of $R$ follow both from the Jacobi identity of $\mathfrak{g}$.

Lemma 1. Let $V$ be a supervector space endowed with a scalar superproduct $\langle\cdot, \cdot\rangle$ and $R$ any algebraic curvature tensor of type $\mathfrak{D} \mathfrak{s p}(V)$. Then $R$ is symmetric in pairs:

$$
\langle R(X, Y) Z, W\rangle=(-1)^{(\widetilde{X}+\widetilde{Y})(\widetilde{Z}+\widetilde{W}}\langle R(Z, W) X, Y\rangle, \quad X, Y, Z, W \in V
$$


Proof. We calculate

$$
\begin{aligned}
&\langle R(X, Y) Z, W\rangle \\
&=-\left\langle(-1)^{\widetilde{X}(\widetilde{Y}+\widetilde{Z})} R(Y, Z) X+(-1)^{\widetilde{Z}(\widetilde{X}+\widetilde{Y})} R(Z, X) Y, W\right\rangle \\
&=\langle X, R(Y, Z) W\rangle+(-1)^{\widetilde{X}(\widetilde{Y}+\widetilde{Z})}\langle Y, R(Z, X) W\rangle \\
&=-\left\langle X,(-1)^{\widetilde{Y}(\widetilde{Z}+\widetilde{W})} R(Z, W) Y+(-1)^{\widetilde{W}(\widetilde{Y}+\widetilde{Z})} R(W, Y) Z\right\rangle \\
&-(-1)^{\widetilde{X}(\widetilde{Y}+\widetilde{Z})}\left\langle Y,(-1)^{\widetilde{Z}(\widetilde{X}+\widetilde{W})} R(X, W) Z+(-1)^{\widetilde{W}(\widetilde{Z}+\widetilde{X})} R(W, Z) X\right\rangle \\
&= 2(-1)^{(\widetilde{X}+\widetilde{Y})(\widetilde{Z}+\widetilde{W})}\langle R(Z, W) X, Y\rangle \\
&+(-1)^{\widetilde{W}(\widetilde{Y}+\widetilde{Z})}\left((-1)^{\widetilde{X}(\widetilde{W}+\widetilde{Y})}\langle R(W, Y) X, Z\rangle+\langle R(X, W) Y, Z\rangle\right) \\
&= 2(-1)^{(\widetilde{X}+\widetilde{Y})(\widetilde{Z}+\widetilde{W})}\langle R(Z, W) X, Y\rangle-(-1)^{\widetilde{W} \widetilde{Z}+\widetilde{Y} \widetilde{X}}\langle R(Y, X) W, Z\rangle \\
&= 2(-1)^{(\widetilde{X}+\widetilde{Y})(\widetilde{Z}+\widetilde{W})}\langle R(Z, W) X, Y\rangle-\langle R(X, Y) Z, W\rangle .
\end{aligned}
$$

This proves (7).

Let us now check that (7) implies (5):

$$
\begin{aligned}
(-1)^{\widetilde{X} \widetilde{Y}}\langle Y,[X, B]\rangle_{\mathfrak{m}} & =-(-1)^{\widetilde{B}(\widetilde{X}+\widetilde{Y})}\langle[B, X], Y\rangle_{\mathfrak{m}} \\
& =(-1)^{(\widetilde{Z}+\widetilde{W})(\widetilde{X}+\widetilde{Y})}\langle R(Z, W) X, Y\rangle_{\mathfrak{m}} \\
& \stackrel{(7)}{=}\langle R(X, Y) Z, W\rangle_{\mathfrak{m}}=-\langle[A, Z], W\rangle_{\mathfrak{m}} \\
& =(-1)^{\widetilde{Z} \widetilde{W}}\langle[A, W], Z\rangle_{\mathfrak{m}} .
\end{aligned}
$$

We have shown that equation (4) defines a scalar superproduct $\langle\cdot, \cdot\rangle=\langle\cdot, \cdot\rangle_{€} \oplus$ $\langle\cdot, \cdot\rangle_{\mathfrak{m}}$ on $\mathrm{g}$. By construction, this scalar superproduct satisfies (3) if at least one of the three vectors $X, Y, Z$ is in $\mathfrak{m}$. The $\operatorname{ad}_{\mathfrak{f}}$-invariance of $\langle\cdot, \cdot\rangle_{\mathfrak{f}}$ follows from that of $\langle\cdot, \cdot\rangle_{\mathfrak{m}}$ and the Jacobi identity (which implies the ad $\mathrm{d}_{\mathfrak{f}}$-invariance

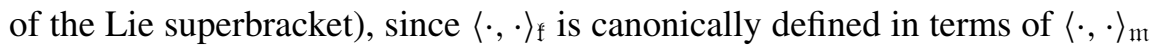
and the Lie superbracket. This finishes the proof of Theorem 6.

Corollary 2. Let $\mathfrak{g}=\mathfrak{g}_{0}+\mathfrak{g}_{1}$ be the Lie superalgebra defined by a four-form $\Omega \in \wedge^{4} E$ satisfying the equation (2). Then there exists a unique $\operatorname{ad}_{\mathfrak{g}_{0}}$-invariant scalar product $\langle\cdot, \cdot\rangle_{0}$ on $\mathrm{g}_{0}$ which extends the $\operatorname{ad}_{\mathfrak{g}_{0}}$-invariant scalar superproduct $\langle\cdot, \cdot\rangle_{1}:=g_{E} \otimes \omega_{H}$ on $\mathfrak{g}_{1}=E \otimes H$ to an $\operatorname{ad}_{\mathfrak{g}}$-invariant scalar superproduct $\langle\cdot, \cdot\rangle=\langle\cdot, \cdot\rangle_{0} \oplus\langle\cdot, \cdot\rangle_{1}$ on g. It is defined by

$$
\left\langle\Omega_{e \wedge e^{\prime}}, \Omega_{e^{\prime \prime} \wedge e^{\prime \prime \prime}}\right\rangle_{0}:=g_{E}\left(\Omega_{e \wedge e^{\prime} e^{\prime \prime}}, e^{\prime \prime \prime}\right) \quad \text { for all } e, e^{\prime}, e^{\prime \prime}, e^{\prime \prime \prime} \in E .
$$

Notice that $g_{E}\left(\Omega_{e \wedge e^{\prime}} e^{\prime \prime}, e^{\prime \prime \prime}\right)=\Omega\left(e, e^{\prime}, e^{\prime \prime}, e^{\prime \prime \prime}\right)$ is the full contraction of $\Omega \in \wedge^{4} E$ with $e \wedge e^{\prime} \wedge e^{\prime \prime} \wedge e^{\prime \prime} \in \wedge^{4} E$ by means of $g_{E}$. Similarly, in the even 
case, $\omega_{E}\left(S_{e e^{\prime}} e^{\prime \prime}, e^{\prime \prime \prime}\right)=S\left(e, e^{\prime}, e^{\prime \prime}, e^{\prime \prime \prime}\right)$ is the full contraction of $S \in S^{4} E$ with $e e^{\prime} e^{\prime \prime} e^{\prime \prime \prime} \in S^{4} E$ by means of $\omega_{E}$.

Proof. It is sufficient to establish the explicit formula for the scalar product, whose existence and uniqueness was proven in Theorem 6 . (Here $\mathfrak{f}=\mathrm{g}_{0}$ and $\mathfrak{m}=\mathfrak{g}_{1}$.) Evaluating (3) for $X=e \otimes h, Y=e^{\prime} \otimes h^{\prime} \in \mathfrak{g}_{1}=E \otimes H$ and $Z=\Omega_{e^{\prime \prime} \wedge e^{\prime \prime \prime}} \in \mathfrak{g}_{0}=\Omega_{E \wedge E}$, we get

$$
\begin{aligned}
\omega_{H}\left(h, h^{\prime}\right)\left\langle\Omega_{e \wedge e^{\prime}}, \Omega_{e^{\prime \prime} \wedge e^{\prime \prime \prime}}\right\rangle_{0} & =-\left\langle e^{\prime} \otimes h^{\prime}, \Omega_{e^{\prime \prime} \wedge e^{\prime \prime \prime}} e \otimes h\right\rangle_{1} \\
& =\omega_{H}\left(h, h^{\prime}\right) g_{E}\left(e^{\prime}, \Omega_{e^{\prime \prime} \wedge e^{\prime \prime \prime}} e\right) \\
& =\omega_{H}\left(h, h^{\prime}\right) g_{E}\left(\Omega_{e \wedge e^{\prime}} e^{\prime \prime}, e^{\prime \prime \prime}\right) .
\end{aligned}
$$

This proves the explicit formula for the scalar superproduct.

\section{Odd symmetric superspaces associated to four-forms}

In this section we will reconsider the Lie superalgebras $\mathfrak{g}=\mathfrak{g}(\Omega)$ constructed in Theorem 5. The symmetric decomposition $\mathfrak{g}=\mathfrak{f}+\mathfrak{m}, \mathfrak{f}:=\mathfrak{g}_{0}, \mathfrak{m}:=\mathfrak{g}_{1}$, can be considered as an infinitesimal model of a symmetric superspace, which is purely odd since $\mathfrak{m}=\mathfrak{g}_{1}$. We will describe its additional invariant special geometric structures.

Since we do not wish to develop, nor to assume, here the theory of Lie supergroups $G$ and their homogeneous superspaces $G / K$, we prefer to keep the discussion on the infinitesimal level. This means that instead of discussing $G / K$ endowed with some geometric structure invariant under the Lie supergroup $G$, we shall only consider the corresponding pairs ( $(\mathfrak{g}, \mathfrak{f})$ of Lie superalgebras endowed with the corresponding $\mathrm{ad}_{\mathfrak{f}}$-invariant linear algebraic data on the vector superspace $\mathfrak{g} / \mathfrak{f}$.

Definition 3. An (effective) infinitesimal homogeneous superspace is a pair $(\mathfrak{g}, \mathfrak{f})$ consisting of a Lie superalgebra $\mathfrak{g}$ and a graded subalgebra $\mathfrak{f} \subset \mathfrak{g}$ such that $\mathfrak{f}$ does not contain any proper ideal of $\mathfrak{g}$. If, moreover, $\mathfrak{g}$ is endowed with a symmetric decomposition $\mathfrak{g}=\mathfrak{f}+\mathfrak{m}$, then $(\mathfrak{g}, \mathfrak{f}, \mathfrak{m})$ is called an infinitesimal symmetric superspace. An odd infinitesimal symmetric space is an infinitesimal symmetric superspace such that $\mathfrak{m}$ is purely odd, i.e. $\mathfrak{m}=\mathfrak{m}_{1}$. Two infinitesimal symmetric superspaces $(\mathfrak{g}, \mathfrak{f}, \mathfrak{m})$ and $\left(\mathfrak{g}^{\prime}, \mathfrak{f}^{\prime}, \mathfrak{m}^{\prime}\right)$ are called isomorphic if there exists an isomorphism of Lie superalgebras $\phi: \mathfrak{g} \rightarrow \mathfrak{g}^{\prime}$ such that $\phi \mathfrak{f}=\mathfrak{f}^{\prime}$ and $\phi \mathfrak{m}=\mathfrak{m}^{\prime}$. The map $\phi: \mathfrak{g} \rightarrow \mathfrak{g}^{\prime}$ is called an isomorphism of infinitesimal symmetric superspaces.

Notice that an infinitesimal symmetric superspace $(\mathfrak{g}, \mathfrak{f}, \mathfrak{m})$ is completely determined by the involutive automorphism $\sigma$ with the eigenspaces

$$
\mathfrak{f}=\{X \in \mathfrak{g} \mid \sigma X=X\} \quad \text { and } \quad \mathfrak{m}=\{X \in \mathfrak{g} \mid \sigma X=-X\} .
$$


The elements of $\mathfrak{m}$ are called infinitesimal transvections. They generate the ideal $\mathfrak{g}_{\mathrm{tr}}:=[\mathfrak{m}, \mathfrak{m}]+\mathfrak{m} \subset \mathfrak{g}$.

Definition 4. Let $(\mathfrak{g}, \mathfrak{f}, \mathfrak{m})$ be an infinitesimal symmetric superspace. It is called minimal if $\mathfrak{f}=[\mathfrak{m}, \mathfrak{m}]$, i.e. $\mathfrak{g}=\mathfrak{g}_{\mathrm{tr}}$. The infinitesimal symmetric space $\left(\mathfrak{g}_{\mathfrak{t r}},[\mathfrak{m}, \mathfrak{m}]\right)$ is called the minimal model of $(\mathfrak{g}, \mathfrak{f}, \mathfrak{m})$. Two infinitesimal symmetric superspaces are called equivalent if their minimal models are isomorphic.

Since we are only interested in infinitesimal symmetric superspaces up to equivalence, we will always assume that $\mathfrak{f}=[\mathfrak{m}, \mathfrak{m}]$.

In the following we will consider infinitesimal symmetric superspaces $(\mathfrak{g}, \mathfrak{f}, \mathfrak{m})$ such that $\mathfrak{m}$ is endowed with some $\operatorname{ad}_{\mathfrak{f}}$-invariant structure, such as a scalar superproduct.

Definition 5. An infinitesimal complex Riemannian symmetric superspace is an infinitesimal symmetric superspace $(\mathfrak{g}, \mathfrak{f}, \mathfrak{m})$ over $C$ equipped with an $\operatorname{ad}_{\mathfrak{f}}$-invariant scalar superproduct $g$ on $\mathfrak{m}$. An infinitesimal pseudoRiemannian symmetric superspace is an infinitesimal symmetric superspace $(\mathfrak{g}, \mathfrak{f}, \mathfrak{m})$ over $\mathrm{R}$ equipped with an $\operatorname{ad}_{\mathfrak{f}}$-invariant scalar superproduct $g$ on $\mathfrak{m}$. If the restriction of the scalar superproduct $g$ to the even part $\mathfrak{m}_{0} \subset \mathfrak{m}=$ $\mathfrak{m}_{0}+\mathfrak{m}_{1}$ is positive definite, then $(\mathfrak{g}, \mathfrak{f}, \mathfrak{m}, g$ ) is called an infinitesimal (real) Riemannian symmetric superspace. An odd infinitesimal Riemannian symmetric space is a real or complex infinitesimal Riemannian symmetric superspace $\left(\mathfrak{g}, \mathfrak{f}, \mathfrak{m}, g\right.$ ) for which $\mathfrak{m}=\mathfrak{m}_{1}$. Two infinitesimal complex Riemannian or pseudo-Riemannian symmetric superspaces $(\mathfrak{g}, \mathfrak{f}, \mathfrak{m}, g)$ and $\left(\mathfrak{g}^{\prime}, \mathfrak{f}^{\prime}, \mathfrak{m}^{\prime}, g^{\prime}\right)$ are called isomorphic if there exists an isomorphism of infinitesimal symmetric superspaces $\phi: \mathfrak{g} \rightarrow \mathfrak{g}^{\prime}$ such that $\phi^{*} g^{\prime}=g$.

With these definitions we can state that the Lie superalgebras $\mathfrak{g}=\mathfrak{g}(\Omega)$ constructed in Theorem 5 define a class of minimal odd infinitesimal symmetric spaces $\left(\mathfrak{g}, \mathfrak{f}=\mathfrak{g}_{0}, \mathfrak{m}=\mathfrak{g}_{1}\right)$ and that the scalar superproduct

$$
g:=\langle\cdot, \cdot\rangle_{1}=g_{E} \otimes \omega_{H}
$$

on $\mathfrak{m}=E \otimes H$ defines on $(\mathfrak{g}, \mathfrak{f}, \mathfrak{m})$ the structure of an odd infinitesimal Riemannian symmetric space if the ground field $\mathrm{K}$ is $\mathrm{R}$ and that of an odd infinitesimal complex Riemannian symmetric space if $K=C$.

In order to speak about the additional special geometric structure of these odd infinitesimal Riemannian symmetric spaces we need yet an other definition.

Definition 6. Let $(\mathfrak{g}, \mathfrak{f}, \mathfrak{m}, g$ ) be an infinitesimal Riemannian (respectively, complex Riemannian) symmetric superspace. A hyper-Kähler (respect- 
ively, complex hyper-Kähler) structure on $(\mathfrak{g}, \mathfrak{f}, \mathfrak{m}, g)$ is a triplet of pairwise anticommuting even complex structures $J_{1}, J_{2}, J_{3}=J_{1} J_{2} \in \operatorname{End}(\mathfrak{m})$ (i.e. $J_{1}^{2}=J_{2}^{2}=J_{3}^{2}=-$ Id) which are skew-symmetric with respect to the scalar superproduct $g$ and which commute with $\operatorname{ad}_{\mathfrak{f}}$. Let $(\mathfrak{g}, \mathfrak{f}, \mathfrak{m}, g)$ be an infinitesimal pseudo-Riemannian symmetric space. A para-hyper-Kähler structure on $(\mathfrak{g}, \mathfrak{f}, \mathfrak{m}, g)$ is a triplet of pairwise anticommuting linear maps $J_{1}, J_{2}, J_{3}=J_{1} J_{2} \in \operatorname{End}\left(g_{1}\right)$ satisfying $J_{1}^{2}=J_{2}^{2}=-J_{3}^{2}=+$ Id which are skew-symmetric with respect to the scalar superproduct $g$ and which commute with $\operatorname{ad}_{f}$.

Theorem 7. Let $\mathrm{K}=\mathrm{C}$. Any infinitesimal complex Riemannian symmetric superspace $(\mathfrak{g}=\mathfrak{g}(\Omega), \mathfrak{f}, \mathfrak{m}, g)$ associated to a four-form $\Omega \in \wedge^{4} E$ satisfying equation (2) admits a complex hyper-Kähler structure. (The scalar superproduct $g$ is defined in (8).) Conversely, any minimal odd infinitesimal complex Riemannian symmetric space which admits a complex hyper-Kähler structure is of this form.

Proof. Let us consider $H=\mathrm{C}^{2}=\mathrm{H}=\operatorname{span}\{1, i, j, k\}$ with the complex structure given by left-multiplication $L_{i}$ with $i$. Then $I_{1}:=R_{i}, I_{2}:=R_{j}$, $I_{3}:=I_{1} I_{2}=-R_{k}$ is a triplet of C-linear anticommuting complex structures on $H$ which are skew-symmetric with respect to the standard complex symplectic structure $\omega_{H}$ of $\mathrm{C}^{2}$. Here $R_{a}$ stands for the right-multiplication by $a \in \mathrm{H}$. The three C-linear operators $J_{\alpha}:=\operatorname{Id}_{E} \otimes I_{\alpha}, \alpha=1,2,3$, on $\mathfrak{m}=\mathfrak{g}_{1}=E \otimes H$ form again a triplet of anticommuting complex structures and are skew-symmetric with respect to the scalar superproduct $g_{E} \otimes \omega_{H}$ on the purely odd vector superspace $\mathfrak{g}_{1}$. Moreover, they are obviously invariant under $\operatorname{ad}_{\mathfrak{f}} \mid \mathfrak{m} \subset \mathfrak{G D}(E) \otimes$ $\operatorname{Id}_{H}$. This proves that $(\mathfrak{g}=\mathfrak{g}(\Omega), \mathfrak{f}, \mathfrak{m}, g)$ admits a complex hyper-Kähler structure.

Now we prove the converse. Let $(\mathfrak{g}, \mathfrak{f}, \mathfrak{m}, g$ ) be any minimal odd infinitesimal complex Riemannian symmetric space which admits a complex hyperKähler structure $\left(J_{1}, J_{2}, J_{3}\right)$. We wish to show that it is defined by a fourform $\Omega$ satisfying equation (2). Notice that by minimality $\mathfrak{f}=[\mathfrak{m}, \mathfrak{m}] \subset \mathfrak{g}_{0}$. Since $\mathfrak{m} \subset \mathfrak{g}_{1}$, this implies that $\mathfrak{f}=\mathfrak{g}_{0}$ and $\mathfrak{m}=\mathfrak{g}_{1}$. Let us denote by $Q \subset \mathfrak{D} \mathfrak{S p}(\mathfrak{m}) \cong \mathfrak{S p}\left(\mathrm{C}^{2 n}\right)$ the Lie algebra generated by the $g$-skew-symmetric endomorphisms $J_{\alpha}$. The action of $Q \cong \mathfrak{S l}(2, \mathrm{C})$ defines an identification $\mathfrak{m}=E \otimes H$ of $(\mathfrak{m}, g)$ with the tensor product of a complex Euclidean vector space $\left(E, g_{E}\right)$ and a two-dimensional complex symplectic vector space $\left(H=\mathrm{C}^{2}, \omega_{H}\right)$ such that $g=g_{E} \otimes \omega_{H}$ and $Q=\operatorname{Id}_{E} \otimes \mathfrak{s p}(H)$.

Let $R$ denote the algebraic curvature tensor of type $\mathfrak{D} \mathfrak{s p}(\mathfrak{m}) \cong \mathfrak{s p}\left(\mathrm{C}^{2 n}\right)$ defined by the equation (6). The operators $R(X, Y), X, Y \in \mathfrak{m}$, commute with the three complex structures $J_{\alpha}$ on $\mathfrak{m}$, by the $\operatorname{ad}_{\mathfrak{f}}$-invariance of the $J_{\alpha}$. This shows that $R: \mathfrak{m} \times \mathfrak{m} \rightarrow \mathfrak{D} \mathfrak{S p}(\mathfrak{m})$ takes values in $\mathfrak{G D}(E) \otimes \operatorname{Id}_{H} \cong \wedge^{2} E$, which 
is the centralizer of $Q$ in $\mathfrak{D} \mathfrak{s} \mathfrak{p}(\mathfrak{m})=\mathfrak{s p}(E \otimes H)$.

Using the fact that the $J_{\alpha}$ are skew-symmetric with respect to $g$ and Lemma 1, it follows that $R: \mathfrak{m} \times \mathfrak{m} \rightarrow \wedge^{2} E$ is invariant with respect to the Lie algebra $Q$. Finally, using the super skew-symmetry of $R(X, Y)$ in $X$ and $Y$, we can interpret $R$ as an $\mathfrak{s p}(H)$-equivariant linear map

$$
R: S^{2} E \otimes S^{2} H+\wedge^{2} E \otimes \wedge^{2} H \rightarrow \wedge^{2} E .
$$

Now Schur's Lemma implies that $R$ vanishes on the first summand $S^{2} E \otimes S^{2} H$, since $S^{2} H$ is a non-trivial irreducible $\mathfrak{s p}(H)$-module. More explicitely, this means that for $X=e \otimes h, Y=e^{\prime} \otimes h^{\prime}, Z=e^{\prime \prime} \otimes h^{\prime \prime} \in \mathfrak{m}=E \otimes H$, we have

$$
[[X, Y], Z]=-R(X, Y) Z=\omega_{H}\left(h, h^{\prime}\right) \Omega\left(e \wedge e^{\prime}\right) e^{\prime \prime} \otimes h^{\prime \prime},
$$

where $\Omega$ is a linear map

$$
\wedge^{2} E \rightarrow \mathfrak{S O}(E) \cong \wedge^{2} E \cong \wedge^{2} E^{*} .
$$

It can be considered as an element $\Omega \in \wedge^{2} E^{*} \otimes \wedge^{2} E^{*}$.

Now it is sufficient to show that $\Omega$ is completely skew-symmetric, i.e. $\Omega \in \wedge^{4} E^{*} \cong \wedge^{4} E$. The symmetry in pairs of $R$, see Lemma 1, implies the symmetry in pairs of $\Omega$, i.e. $\Omega \in S^{2} \wedge^{2} E^{*}$. The (super) Jacobi identity for $X=e \otimes h, Y=e^{\prime} \otimes h^{\prime}, Z=e^{\prime \prime} \otimes h^{\prime \prime} \in \mathfrak{m}=E \otimes H$ reads

$$
\begin{aligned}
& 0= {[[X, Y], Z]+[[Y, Z], X]+[[Z, X], Y] } \\
&=\omega_{H}\left(h, h^{\prime}\right) \Omega\left(e \wedge e^{\prime}\right) e^{\prime \prime} \otimes h^{\prime \prime}+\omega_{H}\left(h^{\prime}, h^{\prime \prime}\right) \Omega\left(e^{\prime} \wedge e^{\prime \prime}\right) e \otimes h \\
&+\omega_{H}\left(h^{\prime \prime}, h\right) \Omega\left(e^{\prime \prime} \wedge e\right) e^{\prime} \otimes h^{\prime} .
\end{aligned}
$$

Choosing $\left(h, h^{\prime}, h^{\prime \prime}\right)$ such that $\omega_{H}\left(h, h^{\prime}\right)=1$ and $h^{\prime \prime}=h$, we get

$$
0=\Omega\left(e \wedge e^{\prime}\right) e^{\prime \prime}-\Omega\left(e^{\prime} \wedge e^{\prime \prime}\right) e,
$$

i.e. the invariance of $\Omega$ under cyclic permutations in the first three arguments. Since $\Omega$ is skew-symmetric in the first two arguments this shows that $\Omega \in$ $\wedge^{3} E^{*} \otimes E^{*}$ and hence $\Omega \in S^{2} \wedge^{2} E^{*} \cap \wedge^{3} E^{*} \otimes E^{*}=\wedge^{4} E^{*}$, as claimed. This proves the theorem.

Remark. Suppose that $E$ is endowed with a quaternionic structure $j_{E}$ which satisfies $j_{E}^{*} g_{E}=\bar{g}_{E}$ and $j_{E}^{*} \Omega=\bar{\Omega}$. Then the odd infinitesimal symmetric space $(\mathfrak{g}=\mathfrak{g}(\Omega), \mathfrak{f}, \mathfrak{m})$ admits a $C$-antilinear automorphism $\rho$ with the following properties:

1) $\left(\mathfrak{g}^{\rho}, \mathfrak{f}^{\rho}, \mathfrak{m}^{\rho}\right)$ is a (real) odd infinitesimal symmetric space. 
2) $g$ is real-valued on the fixed point set $\mathfrak{m}^{\rho}$ of $\left.\rho\right|_{\mathfrak{m}}$, defining on $\left(\mathfrak{g}^{\rho}, \mathfrak{f}^{\rho}, \mathfrak{m}^{\rho}\right)$ the structure of an odd infinitesimal Riemannian symmetric space. The scalar superproduct, denoted by $g^{\rho}$, is the restriction of $g$ to $\mathrm{m}^{\rho}$.

3) The three complex structures $J_{\alpha}, \alpha=1,2,3$, commute with $\left.\rho\right|_{\mathfrak{m}}$ and, hence, define on $\left(\mathfrak{g}^{\rho}, \mathfrak{f}^{\rho}, \mathfrak{m}^{\rho}, g^{\rho}\right)$ a (real) hyper-Kähler structure.

Moreover, any minimal odd infinitesimal Riemannian symmetric space endowed with a hyper-Kähler structure is obtained in this way.

Theorem 8. Let $\mathrm{K}=\mathrm{R}$. Any odd infinitesimal Riemannian symmetric space $\left(\mathfrak{g}=\mathfrak{g}(\Omega), \mathfrak{f}, \mathfrak{m}, g=g_{E} \otimes \omega_{H}\right)$ associated to $\Omega \in \wedge^{4} E$ satisfying equation (2) admits a para-hyper-Kähler structure. Conversely, any minimal odd infinitesimal Riemannian symmetric space, which admits a para-hyperKähler structure is obtained in this way.

Proof. Let us consider the three pairwise anticommuting linear maps

$$
I_{1}=\left(\begin{array}{rr}
1 & 0 \\
0 & -1
\end{array}\right), \quad I_{2}=\left(\begin{array}{ll}
0 & 1 \\
1 & 0
\end{array}\right), \quad I_{3}=I_{1} I_{2}=\left(\begin{array}{rr}
0 & 1 \\
-1 & 0
\end{array}\right)
$$

on $H=\mathrm{R}^{2}$. They satisfy $I_{1}^{2}=I_{2}^{2}=-I_{3}^{2}=\mathrm{Id}$ and are skew-symmetric with respect to the standard symplectic structure $\omega_{H}$ on $H=\mathrm{R}^{2}$. The operators $J_{\alpha}:=\operatorname{Id}_{E} \otimes I_{\alpha}, \alpha=1,2,3$, on $\mathfrak{m}=\mathfrak{g}_{1}=E \otimes H$ satisfy $J_{1}^{2}=J_{2}^{2}=-J_{3}^{2}=$ Id and are skew-symmetric with respect to the scalar superproduct (8). They are obviously invariant under $\operatorname{ad}_{\mathfrak{f}} \mid \mathfrak{m} \subset \mathfrak{S D}(E) \otimes \operatorname{Id}_{H}$.

The converse can be proven along the lines of the previous proof.

\section{Some classes of solutions}

Finally, we construct a large family of solutions to the system (2):

THEOREM 9. Let $E_{0} \subset\left(E, g_{E}\right)$ be an isotropic subspace and $\Omega \in \wedge^{4} E_{0} \subset$ $\wedge^{4} E$. Then $\Omega$ solves (2) and the even part $g_{0}$ of the corresponding Lie superalgebra $\mathfrak{g}=\mathfrak{g}_{0}+\mathfrak{g}_{1}$ is Abelian. In fact more is true, namely $A B=0$ for all $A, B \in \mathfrak{g}_{0} \subset \mathfrak{S D}(E)$, when considered as endomorphisms of $E$. Moreover, any solution of (2) for which $\mathrm{g}_{0}$ is Abelian is of this form.

Proof. Let $E_{0} \subset E$ be an isotropic subspace and $\Omega \in \wedge^{4} E_{0}$. Then $\wedge^{2} E_{0} \subset$ $\wedge^{2} E \cong \mathfrak{S} \mathfrak{D}(E)$ is an Abelian Lie subalgebra with the property that $A B=0$ for all $A, B \in \wedge^{2} E_{0}$ and $g_{0}=\Omega_{E \wedge E} \subset \wedge^{2} E_{0}$. Thus for all $x, y, z, w, u \in E$ we have

$$
\begin{aligned}
\left(\Omega_{x \wedge y} \cdot \Omega\right)_{z \wedge w} u & =\left[\Omega_{x \wedge y}, \Omega_{z \wedge w}\right] u-\Omega_{\left(\Omega_{x \wedge y} z\right) \wedge w} u-\Omega_{z \wedge\left(\Omega_{x \wedge y} w\right)} u \\
& =-\Omega_{\left(\Omega_{x \wedge y} z\right) \wedge w} u-\Omega_{z \wedge\left(\Omega_{x \wedge y} w\right)} u \\
& =-\Omega_{w \wedge u} \Omega_{x \wedge y} z-\Omega_{u \wedge z} \Omega_{x \wedge y} w=0 .
\end{aligned}
$$


This shows that $\Omega$ is a solution of (2) with the claimed properties.

Now let $\Omega$ be a solution of (2) for which $\mathfrak{g}_{0}=\Omega_{E \wedge E}$ is Abelian. This means that for all $x, y, z, w, u \in E$ we have

$$
\begin{aligned}
0=\left(\Omega_{x \wedge y} \cdot \Omega\right)_{z \wedge w} u & =\left[\Omega_{x \wedge y}, \Omega_{z \wedge w}\right] u-\Omega_{\left(\Omega_{x \wedge y} z\right) \wedge w} u-\Omega_{z \wedge\left(\Omega_{x \wedge y} w\right)} u \\
& =0-\Omega_{w \wedge u} \Omega_{x \wedge y} z-\Omega_{u \wedge z} \Omega_{x \wedge y} w \\
& =-\Omega_{x \wedge y} \Omega_{w \wedge u} z-\Omega_{x \wedge y} \Omega_{u \wedge z} w=-2 \Omega_{x \wedge y} \Omega_{z \wedge w} u .
\end{aligned}
$$

Now we can conclude that the support

$$
\Sigma_{\Omega}:=\operatorname{span}\left\{\Omega_{x \wedge y} z \mid x, y, z \in E\right\} \subset E
$$

of $\Omega$ is isotropic. In fact, for all $x, y, z, w, u, v \in E$ we have

$$
g_{E}\left(\Omega_{x \wedge y} u, \Omega_{z \wedge w} v\right)=-g_{E}\left(u, \Omega_{x \wedge y} \Omega_{z \wedge w} v\right)=0 .
$$

This finishes the proof of the theorem, since any four-form $\Omega \in \wedge^{4} E$ satisfies $\Omega \in \wedge^{4} \Sigma_{\Omega}$.

Similarly, one can prove the following theorem, which implies the classification of simply connected complex hyper-Kähler, para-hyper-Kähler and pseudo-hyper-Kähler symmetric spaces with Abelian holonomy group.

THEOREM 10. With the same notation as in the introduction, let $E_{0} \subset$ ( $E, \omega_{E}$ ) be an isotropic subspace and $S \in S^{4} E_{0} \subset S^{4} E$. Then $S$ solves (1) and the even part $\mathrm{g}_{0}$ of the corresponding Lie algebra $\mathfrak{g}=\mathfrak{g}_{0}+\mathfrak{g}_{1}$ is Abelian. In fact more is true, namely $A B=0$ for all $A, B \in \mathfrak{g}_{0} \subset \mathfrak{s} \mathfrak{D}(E)$. Moreover, any solution of (1) for which $\mathfrak{g}_{0}$ is Abelian is of this form.

The following example shows that there exists solutions of (2) for which $\mathfrak{g}_{0}$ is not nilpotent but only solvable.

Example 1. Consider $E=\mathrm{K}^{6}$ endowed with the scalar product $g_{E}$ defined by $g_{E}\left(p_{i}, p_{j}\right)=g_{E}\left(q_{i}, q_{j}\right)=0$ and $g_{E}\left(p_{i}, q_{j}\right)=\delta_{i j}$, with respect to some basis $\left(p_{1}, p_{2}, p_{3}, q_{1}, q_{2}, q_{3}\right)$ of $E$. Then

$$
\Omega=p_{1} \wedge p_{2} \wedge p_{3} \wedge q_{3}
$$

is a solution of (2) and

$$
\mathrm{g}_{0}=\Omega_{E \wedge E}=\operatorname{span}\left\{p_{1} \wedge p_{2}, p_{1} \wedge p_{3}, p_{2} \wedge p_{3}, p_{1} \wedge q_{3}, p_{2} \wedge q_{3}, p_{3} \wedge q_{3}\right\}
$$

is a 3-step solvable Lie algebra, which is not nilpotent. This example can be generalized as follows: 
THEOREM 11. Consider $E=\mathrm{K}^{n+4}$ endowed with a scalar product $g_{E}$ and an orthogonal decomposition $E=\mathrm{K}^{4}+E^{\prime}$. Assume that $\mathrm{K}^{4}$ admits a basis $\left(p_{1}, p_{2}, q_{1}, q_{2}\right)$ such that $g_{E}\left(p_{i}, p_{j}\right)=g_{E}\left(q_{i}, q_{j}\right)=0$ and $g_{E}\left(p_{i}, q_{j}\right)=\delta_{i j}$. (Such a basis does always exist if $\mathrm{K}=\mathrm{C}$.)

(i) For any $A \in \wedge^{2} E^{\prime} \cong \mathfrak{S} \mathfrak{D}\left(E^{\prime}\right) \subset \mathfrak{S} \mathfrak{D}(E)$ the four-form

$$
\Omega=p_{1} \wedge p_{2} \wedge A
$$

is a solution of (2) for which $\mathrm{g}_{0}$ is a solvable Lie algebra.

(ii) The Lie algebra $\mathfrak{g}_{0}$ is at most 3-step solvable. It is precisely 3-step solvable if $A^{4} \neq 0$.

(iii) The Lie algebra $\mathrm{g}_{0}$ is nilpotent if and only if $A$ is nilpotent. More precisely, $\mathrm{g}_{0}$ is $k$-step nilpotent if and only if $A^{k} \neq 0$ and $A^{k+1}=0$.

Proof. Let us first calculate $\mathfrak{f}=\mathfrak{g}_{0}=\Omega_{E \wedge E}$ :

$$
\mathfrak{f}=\operatorname{span}\left\{p_{1} \wedge p_{2}, A\right\}+p_{1} \wedge A E+p_{2} \wedge A E .
$$

From this, one can easily check that $\mathfrak{f} \cdot \Omega=0$. Next we calculate the lower central series

$$
\mathscr{D}_{k} \mathfrak{f}=\left[\mathfrak{f}, \mathscr{D}_{k-1} \mathfrak{f}\right]=p_{1} \wedge A^{k+1} E+p_{2} \wedge A^{k+1} E+p_{1} \wedge p_{2} g_{E}\left(E, A^{k+1} E\right),
$$

where $k \geq 1$ and $\mathscr{D}_{0} \mathfrak{f}:=\mathfrak{f}$. (Notice that $g_{E}\left(E, A^{k+1} E\right)=\mathrm{K}$ if $A^{k+1} \neq 0$.) This shows that $f$ is $k$-step nilpotent if and only if $A^{k} \neq 0$ and $A^{k+1}=0$. Finally,

$$
\left[\mathscr{D}_{1} \mathfrak{f}, \mathscr{D}_{1} \mathfrak{f}\right]=p_{1} \wedge p_{2} g_{E}\left(E, A^{4} E\right),
$$

and, hence, $\mathfrak{f}$ is at most 3 -step solvable.

The following example shows that there exists also solutions of (2) for which $\mathrm{g}_{0}$ is semisimple.

EXAMPLE 2. If $\operatorname{dim} E=4$, any four-form $\Omega \in \wedge^{4} E \cong \mathrm{K}$ is a solution of (2) and, if $\Omega \neq 0$, we have that $\mathfrak{g}_{0}=\mathfrak{s} \mathfrak{D}(E)$. The corresponding Lie superalgebra $\mathrm{g}$ is simple and belongs to the orthosymplectic series.

It is a challenging problem to determine the general solution of (2).

\section{REFERENCES}

1. Alekseevsky, D. V., and Cortés, V., Classification of indefinite hyper-Kähler symmetric spaces, Asian J. Math. 5 (2001), no. 4, 663-684.

2. Alekseevsky, D. V., Blažić, N., Cortés, V., and Vukmirović, S., A class of Osserman spaces, J. Geom. Phys. 53 (2005), 345-353. 
3. Benayadi, S., Quadratic Lie superalgebras with the completely reducible action of the even part on the odd part, J. Algebra 223 (2000), no. 1, 344-366.

4. Besse, A., Einstein Manifolds, Ergeb. Math. Grenzgeb., 1984.

5. Bordemann, M., Nondegenerate invariant bilinear forms on nonassociative algebras, Acta Math. Univ. Comenian. (N.S.) 66 (1997), no. 2, 151-201.

6. Cahen, M., and Parker, M., Pseudo-Riemannian symmetric spaces, Mem. Amer. Math. Soc. 24 (1980), no. 229, iv+108 pp.

7. Kath, I., and Olbrich, M., New examples of indefinite hyper-Kähler symmetric spaces, math.DG/0503220.

8. Kostant, B., A cubic Dirac operator and the emergence of Euler number multiplets of representations for equal rank subgroups, Duke Math. J. 100 (1999), no. 3, 447-501.

9. Kostant, B., The Weyl algebra and the structure of all Lie superalgebras of Riemannian type, Transform. Groups 6 (2001), no. 3, 215-226.

10. Kostant, B., and Sternberg, S., Symplectic reduction, BRS cohomology, and infinite-dimensional Clifford algebras, Ann. Physics 176 (1987), no. 1, 49-113.

11. Medina, A., and Revoy, Ph., Algèbres de Lie et produit scalaire invariant, Ann. Sci. École Norm. Sup. (4) 18 (1985), no. 3, 553-561.

DEPARTMENT FÜR MATHEMATIK

SCHWERPUNKT ANALYSIS UND DIFFERENTIALGEOMETRIE

UND

ZENTRUM FÜR MATHEMATISCHE PHYSIK

UNIVERSITÄT HAMBURG

BUNDESSTRAßE

20146 HAMBURG

GERMANY

E-mail: cortes@math.uni-hamburg.de 